\title{
Article \\ A Low Dark Current 160 dB Logarithmic Pixel with Low Voltage Photodiode Biasing
}

\author{
Alessandro Michel Brunetti ${ }^{1, *,+}$ and Bhaskar Choubey ${ }^{2,3}$ \\ 1 Department of Engineering Science, University of Oxford, Parks Road, Oxford OX1 3PJ, UK \\ 2 Analogue Circuits and Image Sensors, Universität Siegen, Hölderlinstr. 3, 57076 Siegen, Germany; \\ bhaskar.choubey@uni-siegen.de \\ 3 Microelectronic Intelligence, Fraunhofer Institute of Microelectronics Circuits and Systems, \\ 47057 Duisburg, Germany \\ * Correspondence: ale@absensing.com \\ + Current Address: Absensing LTD., Oxford, UK.
}

Citation: Brunetti, A.M.; Choubey, B. A Low Dark Current $160 \mathrm{~dB}$ Logarithmic Pixel with Low Voltage Photodiode Biasing. Electronics 2021, 10, 1096. https://doi.org/10.3390/ electronics10091096

Academic Editors: Marco Lanuzza, Raffaele De Rose and Sebastiano Strangio

Received: 31 March 2021

Accepted: 3 May 2021

Published: 7 May 2021

Publisher's Note: MDPI stays neutral with regard to jurisdictional claims in published maps and institutional affiliations.

Copyright: () 2021 by the authors. Licensee MDPI, Basel, Switzerland. This article is an open access article distributed under the terms and conditions of the Creative Commons Attribution (CC BY) license (https:// creativecommons.org/licenses/by/ $4.0 /)$.

\begin{abstract}
Extending CMOS Image Sensors' dynamic range is of fundamental importance in applications, such as automotive, scientific, or X-ray, where a broad variation of incoming light should be measured. The typical logarithmic pixels suffer from poor performance under low light conditions due to a leakage current, usually referred to as the dark current. In this paper, we propose a logarithmic pixel design capable of reducing the dark current through low-voltage photodiode biasing, without introducing any process modifications. The proposed pixel combines a high dynamic range with a significant improvement in the dark response compared to a standard logarithmic pixel. The reported experimental results show this architecture to achieve an almost $35 \mathrm{~dB}$ improvement at the expense of three additional transistors, thereby achieving an unprecedented dynamic range higher than $160 \mathrm{~dB}$.
\end{abstract}

Keywords: CMOS; sub-threshold; pixel; image sensors; logarithmic; high dynamic range

\section{Introduction}

Technological advances in CMOS image sensors (CIS) have boosted their utilization in a variety of applications. In particular, CIS are widely employed in battery powered devices, such as smartphones or surveillance cameras, which have boosted interest in the design of low-power circuits to reduce battery consumption [1-5]. At a system level, the effort to reduce power consumption has been conducted in various directions. For example, research has been focused on harvesting energy from the environment [6,7]; however, energy harvesting systems still require low-power CIS. Furthermore, readout circuits, including analog-to-digital converters (ADC) are usually power hungry, and a significant effort has been undertaken to reduce ADC consumption [1,3,8]. Alternative readout schemes have been proposed to reduce power consumption [9], while other analog encoding voltage techniques have been introduced to boost dynamic range (DR) [10]. The need for high DR (HDR) imaging is mostly driven by automotive applications, where the light conditions can vary significantly [11]. Alternative applications, such as scientific imaging, may benefit from an HDR sensor. Several approaches have been proposed to increase the DR [12-14]. However, most of these solutions require a high number of samples, several transistors per pixel and/or computationally demanding post-processing. One candidate pixel architecture that combines HDR and low power is the logarithmic pixel, which exploits transistor subthreshold operation [15-17]. Unfortunately, in low light conditions, their sensitivity to light is limited [18] as in other CMOS pixels. The inherent high leakage current linked with CMOS processes is one of the principal causes of this degradation. This leakage current is also referred to as the "dark current" for its presence in the absence of light. The dark current is due to defects in the photodiode and at the boundaries and is 
mainly related to the manufacturing process. Therefore, the techniques to reduce the dark current also usually involve process modifications, generally by introducing either barriers to avoid the interaction with the source of the defects or by curing the defects, for example, with annealing [19-21]. Unfortunately, any technique that involves process modification is costly to implement and is generally of limited availability to circuit designers. Other techniques based on circuit implementations have been presented. The dark current has been proven to be reduced by applying a negative offset operation on the gate of the transfer gate transistor [22] or by clever layout techniques, which also improve the light response [23,24]. Furthermore, integrating CMOS Active Pixel Sensors have a limited dynamic range of 2-3 decades. This means that any significant improvement at the dark end of the response may lead to poor performance in the brighter regions. Therefore, an HDR sensor is required to fully utilize the advantages offered by reducing the dark current. A low dark current logarithmic pixel was previously reported [25] where a theoretical analysis and simulation mismatch were conducted; however, no experimental evidence was provided. Experimental methods have been proposed to correct the mismatch in logarithmic pixels by modeling and calibration [26]. In this paper, we report definitive proof of operation of a single, low, dark current pixel, which is manufactured in a CMOS technology and compared to a standard logarithmic pixel. The subthreshold logarithmic pixel architecture is intended for large formats and meets the needs of scientific imaging where low power, low dark current and wide dynamic range may be employed and where the demand of speed or pixel scaling is limited.

This paper is organized as follows. In Section 2, we first present the physical foundation of our approach and the classic 3T logarithmic pixel. We also present the proposed approach with low voltage bias and enhanced low light operation. Section 3 presents the experimental results, while Section 4 concludes the paper.

\section{Methodology}

In CMOS image sensors, the dark current is mainly attributed to two different sources, in addition to any leakage due to surface or periphery defects. The first is the current injection-diffusion that originates from the random movement of carriers and depends on their concentration. The second is the thermal carrier generation-recombination described by the Shockley-Read-Hall equation for generation-recombination and directly depends on the applied voltage across the photodiode depletion layer. In CMOS pixels, the photodiode is usually operated in a reverse bias. Figure 1 shows a simulated I-V characteristic of the photodiode at different light intensities and different bias voltages in a typical CMOS process. As can be observed in the figure, the photodiode leakage current is at its minimum value at a zero-voltage bias. When biased with a negative voltage, the dark current increases from this minimal value. Due to this, one may imagine that the dark current can be reduced if the photodiode operates as close as possible to zero volts. In this paper, we show that the logarithmic pixel can exploit a zero-volt operation, trading off against the use of additional transistors.

The strategy used to reduce the dark current is to keep the photodiode voltage close to zero. This is not possible using the standard Active Pixel Sensor, as it has limited dynamic range (DR). Hence, improving the dark current without enhancing the dynamic range will not fully exploit the high light region performance. A logarithmic pixel which converts the input photocurrent to an output logarithmic voltage is the optimum for single-frame recording, with the minimum number of transistors and limited post-processing required. Unfortunately, this pixel is worst affected by the dark current. Figure 2 shows a typical logarithmic pixel where the nMOS load transistor $M_{1}$ is operating in the subthreshold region. $M_{2}$ and $M_{3}$ are the source follower (SF) and the row selector (RS), respectively, PD is the photodiode, $V_{D D}$ is the supply voltage and col output is the column output node. The drain current of transistor $M_{1}$ is logarithmically related to the drain to source voltage as shown in Equation (1). 


$$
V_{S, M 1}=V_{D D}-V_{T H}-n V_{T} \ln \left(\frac{I_{D S, M 1}}{I_{0}}\right)
$$

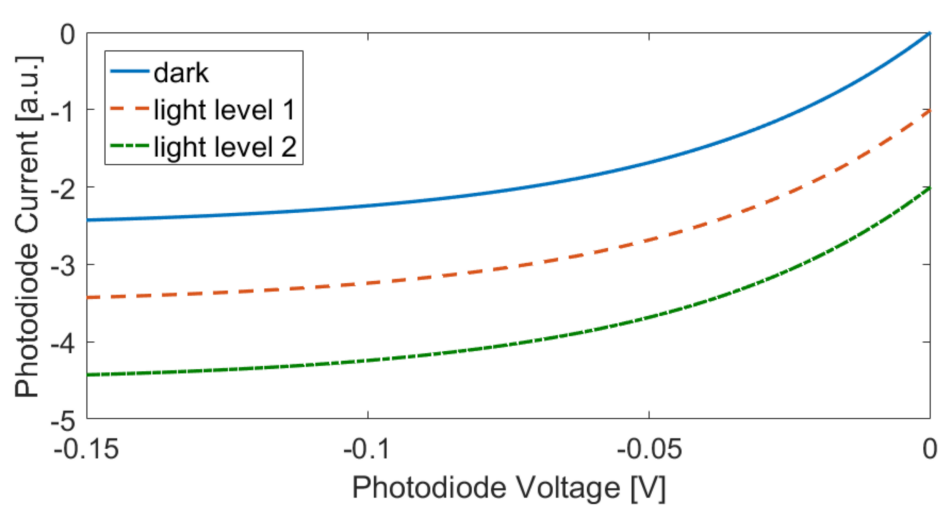

Figure 1. Simulated I-V characteristic of the photodiode for increasing levels of light. The photodiode current is expressed in arbitrary units (a.u.).

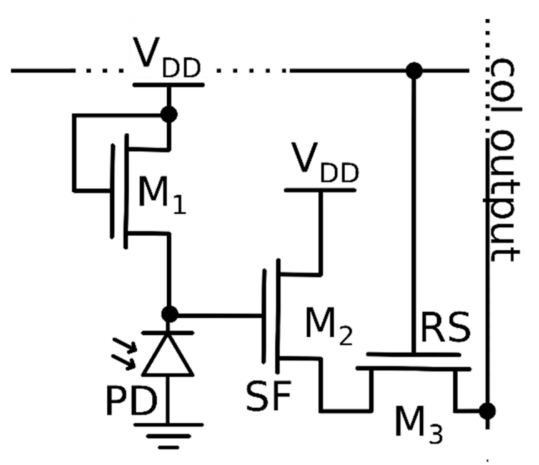

Figure 2. A typical logarithmic pixel with single nMOS load [25].

In Equation (1), $V_{D D}=V_{G M 1}$ is the supply voltage, $V_{T H}$ is the threshold voltage, $V_{T}$ is the thermal voltage, $I_{D S, M 1}$ is the drain current flowing in $M_{1}, I_{0}$ is a characteristic current that defines the current that leaks through the transistor $M_{1}$ and $n$ is the subthreshold slope factor. Ideally, the output voltage is logarithmically proportional to the photogenerated current due to the incoming light. Unfortunately, the dark current produced in the photodiode gets added to the photogenerated current, limiting the sensitivity at the darker end of the pixel response. This effect also limits the gain sensitivity of the pixel. A possible solution to enhance the pixel response is to reduce the dark current. A circuit that implements this technique is presented in the next section.

It is worth noting that the main contribution of a photodiode dark current is related to the voltage applied across the photodiode as presented in Figure 1. The typical bias voltage applied to the diode in a logarithmic pixel is similar to the pixel output. For example, the analogue supply voltage can be $3.3 \mathrm{~V}$ and hence, the output voltage is often comprised between 2.5 and $1.8 \mathrm{~V}$, which generates a significant leakage contribution. A possible solution to reduce the dark current is to force a zero-potential difference across the photodiode. Such an approach is proposed in the pixel shown in Figure 3. While in a standard logarithmic pixel, the output node is connected to the node biasing the photodiode; in the proposed circuit, this is split into two different nodes. In fact, this circuit is able to maintain a nearly zero potential across the photodiode, resulting in minimal leakage current, as the generation-recombination contribution to the dark current is close to zero. In this pixel, transistors $M_{3}$ and $M_{4}$ form a pMOS current mirror, and transistors $M_{2}$ and $M_{1}$ form a nMOS current mirror. 


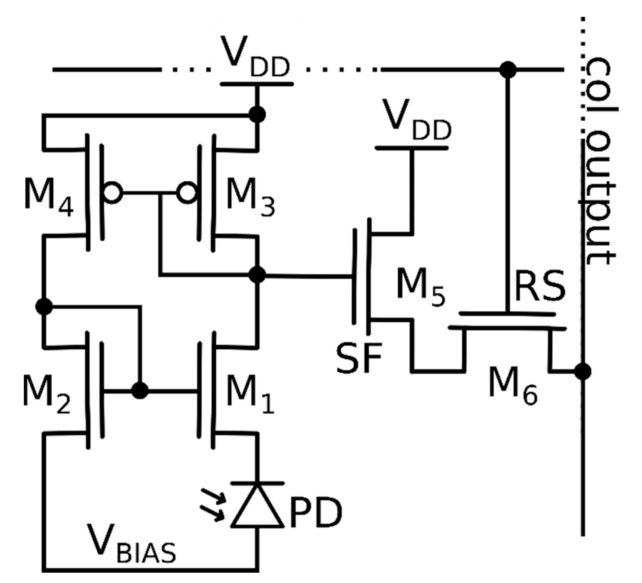

Figure 3. Proposed double-mirrored, low, dark current pixel design. In the proposed pixel design, 3 transistors are added compared to the logarithmic pixel with the purpose of enforcing a zero-voltage potential across the photodiode [25].

The output to the SF $M_{5}$ and to the readout circuitry is taken from the drain of the nMOS $M_{1}$, while the photodiode is connected to its source. In this pixel, the transistors are usually operating in subthreshold due to the low photocurrent produced in typical lighting conditions and the diode size used. The idea of the circuit is to copy the photogenerated current in the two branches of the circuit consisting of $M_{1}, M_{3}$ and $M_{2}, M_{4}$, respectively. When the same current flows in transistors $M_{1}$ and $M_{2}$, these will have the same gate-source voltage, in turn. The voltage drop on the photodiode can be expressed as the difference between the gate-source voltages of the nMOS couple $M_{2}$ and $M_{1}$ when considering the loop formed by the gate-source of the nMOS mirror pair and the photodiode, and can be written as follows:

$$
V_{P D}=V_{G S, M_{2}}-V_{G S, M_{1}}
$$

where $V_{P D}$ is the cathode-to-anode photodiode voltage and $V_{G S, M 1}$ and $V_{G S, M 2}$ are the gate-source voltages or the transistors $M_{1}$ and $M_{2}$, respectively. In turn, the source voltages depend on the biasing current of each pair and, for a subthreshold operating region, is expressed as follows:

$$
V_{G S, M x}=n V_{T} \ln \left(\frac{I_{D S, M x}}{I_{M x}}\right)+V_{T H}
$$

where $V_{G S, M x}$ is the gate-source voltage of the transistors, $V_{T H}$ is the threshold voltage, $V_{T}$ is the thermal voltage, $I_{D S, M x}$ is the drain current flowing in either $M_{1}$ or $M_{2}$ and $I_{M x 0}$ is a characteristic current that defines the current that leaks through transistors $M_{1}$ and $M_{2}$ and $\mathrm{n}$ is the subthreshold slope factor.

The top part of the circuit consists of a pMOS pair formed by identically sized transistors, $M_{3}$ and $M_{4}$, that replicate the current flowing in the right branch of the circuit into the left one, thereby achieving almost identical currents in both branches. The currents are almost identical, as we are not considering the mismatch of the devices in this analysis as it has been previously reported by the authors [25]. In this case, if the drain-source currents are identical, the gate-source voltages should also be identical, thereby achieving a zero voltage drop across the photodiode. The current flowing in the branch is the sum of the photogenerated current and the dark current. When the photodiode is not exposed to light, only the dark current flows in the pixel. In this case, the output voltage is high; however, in the proposed pixel, this is the source of $\mathrm{M}_{3}$, which is not directly connected to the photodiode as opposed to the logarithmic pixel. Thanks to this feature, the advantage of the proposed pixel compared to the typical logarithmic pixel is that the photodiode voltage is enforced to zero, thereby reducing the dark current. A lower dark current directly translates to a higher sensitivity at the darker end of the response, with a significant increase in the dynamic range in this portion of the pixel response. 
The logarithmic response of the pixel is granted, thanks to the load transistor $M_{3}$, which is a diode-connected pMOS transistor. Equation (1) similarly applies and describes the output of the pixel, apart from the well-known differences between nMOS and pMOS transistors. The transistors $M_{5}$ and $M_{6}$ are the SF and the RS transistor, respectively. $V_{\text {BIAS }}$ is the anode voltage of the photodiode. Depending on the process technology and the size of the transistors, this can either be connected to the ground or the bias voltage $\mathrm{V}_{\mathrm{BIAS}}$. In fact, let us consider the expression of the threshold voltage [27]:

$$
V_{T H}=V_{F B}+2 \psi_{B}+\frac{\sqrt{2 \epsilon_{s i} q N_{a}\left(2 \psi_{B}+V_{B S}\right)}}{C_{o x}}
$$

where $V_{F B}$ is the flat-band voltage, $N_{a}$ is the doping density in the substrate, $C_{o x}$ is the gate oxide capacitance, $\epsilon_{s i}$ is the dielectric constant of the silicon, $q$ is the electron charge and $\psi_{\mathrm{B}}=(K T / q) \log \left(N_{a} / n_{i}\right)$ is the difference between the Fermi potential and the intrinsic potential in the substrate. A change of the body-source voltage will result in a variation of the threshold voltage. In the considered Equations (1)-(3), second-order effects have been ignored, and the change of $\mathrm{V}_{\mathrm{TH}}$ should not affect the behavior of the current mirror. However, considering a more advanced expression of the subthreshold leakage [28]:

$$
I_{D S, \text { subth }}=I_{S} e^{\frac{1}{n} V_{T}\left(V_{G S}-V_{T H 0}-\gamma^{\prime} V_{S}+\eta V_{D S}\right)}
$$

where $I_{S}$ is a factor that depends on the aspect ratio, $V_{T H 0}$ is the zero bias threshold voltage, and $\eta$ is the Drain-Induced Barrier Lowering (DIBL) coefficient. For small values of $V_{B S}$, the body effect is linear and it is represented by the term $\gamma^{\prime} V_{S}$, where $\gamma^{\prime}$ is the linearized body effect coefficient. The subthreshold current is dependent on the $V_{D S}$ voltage. In the double current mirror circuit, transistors $M_{1}$ and $M_{2}$ have different $V_{D S}$ voltages. This difference causes a mismatch between the two IDS currents. In turn, the mismatch between the currents causes the $V_{G S}$ of the current mirror pair to be different, as well. This causes a voltage drop across the photodiode. However, increasing the $V_{B S}$ voltage increases the effect of the first term in the exponential, compared to the second term where the $V_{D S}$ is present. If sufficiently increased, the mismatch between the two currents will be reduced, and the photodiode voltage drop will be reduced as well. Alternatively, it is possible to increase the length of the transistor channels to minimize the DIBL effect. For the purpose of this paper, the transistor channel length was increased to $5 \mu \mathrm{m}$ and the $V_{B I A S}$ voltage was set to ground.

Figure 4 presents the pixel layout of the pixels that are compared in this study. Figure $4 \mathrm{a}$ presents the layout of the logarithmic pixel, while Figure $4 \mathrm{~b}$ shows the layout of the proposed low dark current pixel. The load transistors, $M_{1}$ and $M_{3}$, have been designed with the same sizes, $W=5 \mu \mathrm{m}$, while their length is the minimum length allowed by the technology. The transistors are standard from the LFoundry CIS $110 \mathrm{~nm}$ PDK: low Vt 3.3 V MOS.

The chosen size of the photodiode $(100 \mu \mathrm{m})$ does not allow a representation in-scale of the pixel. However, while in X-ray applications, large pixels are often utilized, in other applications, smaller pixels may be of interest. For this reason, we introduce a scaling model to estimate the fill factor achievable with the proposed design. Let us define $\mathrm{p}$ as the pixel pitch. The total area of the pixel can be then written as $A_{\text {pix }}=p^{2}$. To evaluate the fill factor, we need to evaluate the area of the photodiode. This can be estimated as the difference between the pixel area and the area occupied by the transistors $A_{P D}=A_{\text {pix }}-A_{t r}$. The estimation of the area occupied by the transistors depends on the specific design considered and the design choices; however, for the case in this example, we can consider that the area of the SF and RS transistors is the same in both the logarithmic pixel and the proposed low dark current pixel. If the diffusion-to-diffusion minimum distance is also accounted for, it is possible to estimate the SF and RS area as $0.75 \mu \mathrm{m}^{2}$ $(1.5 \mu \mathrm{m} \times 0.5 \mu \mathrm{m})$. In the logarithmic pixel, the load transistor $M_{1}$ area can be estimated as $2.75 \mu \mathrm{m}^{2}(5.5 \mu \mathrm{m} \times 0.5 \mu \mathrm{m})$, while in the proposed low dark current pixel, the core 
transistor area can be estimated as $11 \mu \mathrm{m}^{2}(5.5 \mu \mathrm{m} \times 0.5 \mu \mathrm{m} \times 4$ transistors). Recalling the expression of the fill factor as the percent ratio between the photodiode area and the pixel area, it is possible to formalize the following:

$$
\begin{aligned}
& F F_{l o g}=100 * \frac{A_{P D l o g}}{A_{\text {pix }}}=100 \times \frac{p^{2}-A_{M 1}-A_{S F, R S}}{p^{2}} \\
& F F_{l d c}=100 * \frac{A_{P D l d c}}{A_{\text {pix }}}=100 \times \frac{p^{2}-4 A_{M}-A_{S F, R S}}{p^{2}}
\end{aligned}
$$

where $A_{P D l o g}$ and $A_{P D l d c}$ are the areas of the logarithmic pixel photodiode and the low dark current pixel photodiode, respectively. $A_{M 1}=A_{M}$ is the area of the load transistors and $A_{S F, R S}$ is the area of the SF and RS transistors.

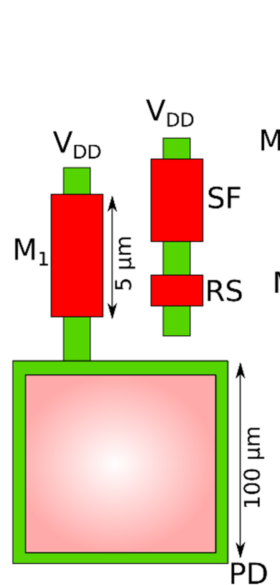

(a)

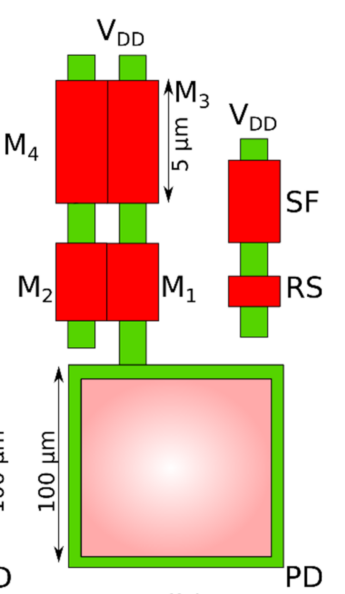

(b)

Figure 4. Pixel layout (not in scale). (a) Logarithmic pixel. (b) Proposed low dark current pixel. The diffusion is in green, the polysilicon is in red and the photodiode sensitive area is in pink.

Figure 5 shows the calculated fill factor of Equations (6) and (7) for the logarithmic pixel and the proposed low dark current pixel, respectively. While for the pixel pitch of choice the difference is negligible, for smaller pixel pitches, the difference in fill factor increases. With LFoundry technology and the chosen transistor parameters, the achievable pixel pitch for the logarithmic pixel is smaller than the proposed low dark current pixel in front side illuminated technologies. However, for scientific applications, the typical pixel pitch is significatively larger, and for pitches above $20 \mu \mathrm{m}$, the difference can be neglected.

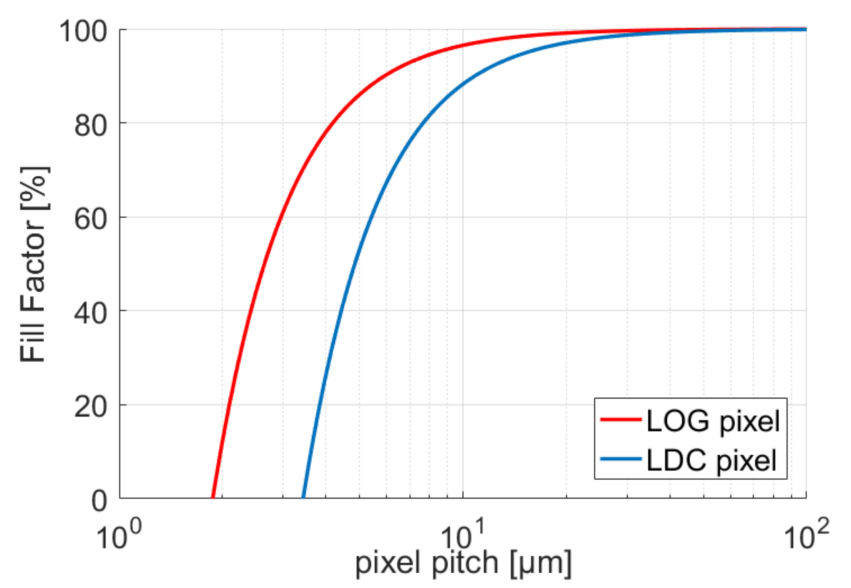

Figure 5. Calculated fill factor in semilogarithmic scale for both the logarithmic pixel (in red) and the proposed low dark current pixel (in blue). 


\section{Experimental Results}

This pixel was manufactured with LFoundry $110 \mathrm{~nm}$ Image Sensor process technology. It was implemented as a single device structure to prove functionality. In order to compare the proposed pixel with the standard logarithmic pixel, both versions have been designed and fabricated. Recalling Figure 3, the load of the proposed pixel consists of a pMOS device. Therefore, the logarithmic pixel designed for this experiment also employs a pMOS as a load. In order to ensure a reliable comparison, both the standard and the proposed logarithmic pixels were connected to a photodiode of the same size $(100 \times 100 \mu \mathrm{m})$. Both were designed close in the die and exposed to the same light generated by a calibrated source during the measurements. The choice of a large pixel of $100 \mu \mathrm{m}$ pitch also helps with the characterization. In fact, the typical dark current values nowadays in CIS are in the range of the $\mathrm{pA} / \mathrm{cm}^{2}$ and may be particularly difficult to measure. A larger photodiode provides a higher current, enabling measurements with common laboratory equipment.

The pixels were measured in a laboratory environment where a calibrated light source was used to shine the light through an integrating sphere to guarantee uniformity. The measurement setup was equipped with a high sensitivity photodiode. The measurement results are shown in Figure 6, where the pixel output voltages are plotted against the measured incoming light intensity. The red diamonds represent the response of the standard logarithmic pixel, while the blue circles represent the response of the proposed low dark current pixel. In addition, the data were fitted with the model presented in Equation (8) in order to estimate the dark current levels. Equation (8) is a model for Equation (1), where the dark current has been made explicit and the parameters simplified, resulting in the following [10]:

$$
y=a+b \ln (x+c),
$$

where $a$ is the offset, $b$ is the gain and $c$ is the dark current contribution of each pixel. The terms $a$ and $b$ are characteristic parameters, depending on the process, temperature and to the readout circuitry. In this experiment, the measurements were conducted at the same temperature and with the same process technology. Therefore, the aforementioned parameters are expected to be similar for both pixels. However, the c-terms are different, as they represent the different levels of the dark current, which, in turn, depend on the bias voltage of the photodiode. Let us define $c_{\log }$ and $c_{\text {ldc }}$, the dark current terms for the logarithmic and low dark current pixel, respectively. The ratio between the $\mathrm{c}$ terms represents the dark current reduction rate. The measured ratio is $c_{l d c} / c_{l o g}=-34.54 \mathrm{~dB}$, which corresponds to a reduction in the dark current by a factor higher than 50. In addition, we can analyze the slope of the pixel responses to determine their sensitivity. Figure 7 shows the slopes of both the logarithmic and low dark current pixels.

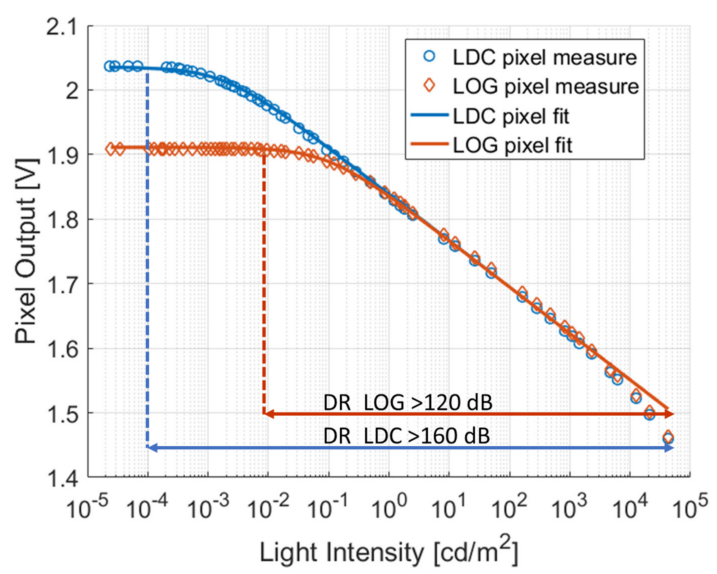

Figure 6. Measured responses of the logarithmic pixel (red diamonds) fitted with the red line compared to the proposed low dark current pixel (blue circles) fitted with the blue line. The dynamic range (DR) is highlighted for both pixel versions. 


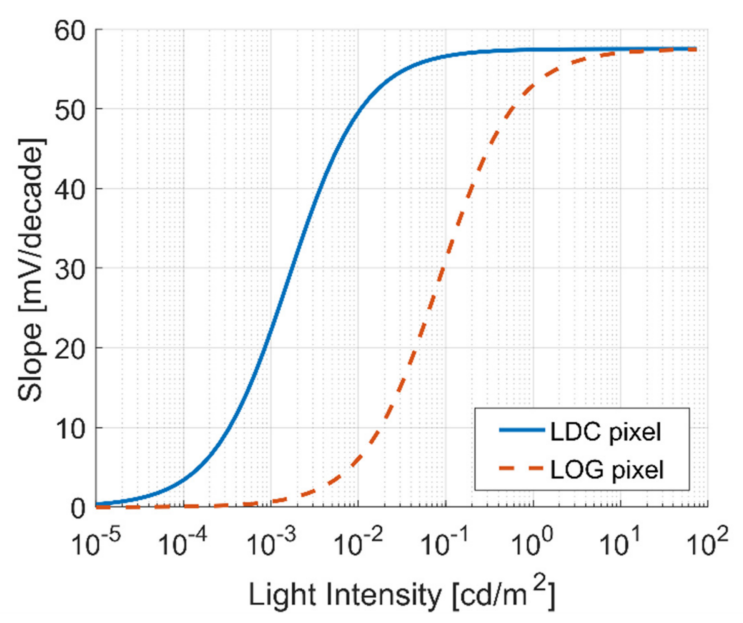

Figure 7. Slope of the measured logarithmic pixel response (red dotted line) compared to the measured proposed low dark current pixel response (blue line).

The slope represents the sensitivity of the pixel as a function of the input light intensity. From this figure, the low dark current pixel exhibits a higher sensitivity for lower values of incoming light compared to the logarithmic pixel. In their normal region of operation, both pixels have a slope of $58 \mathrm{mV} /$ decade. Furthermore, this is guaranteed for a higher range in the proposed pixel. From the analysis conducted in this paper, it is possible to conclude that the proposed low dark current pixel circuit has a wider dynamic range of $\sim 35 \mathrm{~dB}$ at the darker end of the response compared to a logarithmic pixel. The results are summarized in Table 1.

Table 1. Summary of pixel performance.

\begin{tabular}{ccc}
\hline Parameter & LDC Pixel & LOG Pixel \\
\hline Technology & LFoundry $110 \mathrm{~nm}$ CIS process \\
\hline Photodiode pitch & $100 \mu \mathrm{m}$ \\
\hline Photodiode bias & $<5 \mathrm{mV}$ & $2.2-2.6 \mathrm{~V}$ \\
\hline Dynamic Range & $>160 \mathrm{~dB}$ & $>120 \mathrm{~dB}$ \\
\hline $\begin{array}{c}\text { Dark Current Ratio } \\
\text { (LDC/LOG) }\end{array}$ & & $-34.54 \mathrm{~dB}$ \\
\hline
\end{tabular}

\section{Conclusions}

The dark current limits the performance of CMOS image sensors. In this paper, starting from the observation that enforcing a zero potential across the photodiode leads to a dark current reduction, a pixel circuit able to maintain a zero potential across the photodiode was presented. The proposed circuit consists of six transistors, has a logarithmic response and the advantage of reducing the dark current by enforcing a low voltage potential across the photodiode. In this paper, we compared this low dark current pixel to a standard logarithmic pixel. Both pixels were manufactured in LFoundry $110 \mathrm{~nm}$ CIS process technology, using standard low VT $3.3 \mathrm{~V}$ transistors and the same photodiode size of $100 \mu \mathrm{m}$. The proposed pixel is able to maintain a low voltage bias across the photodiode, which results in a significant reduction in dark current. In fact, the dark current improves by $34.5 \mathrm{~dB}$, which directly translates to an increased dynamic range, thanks to the broader response at the darker end of the characteristic. This improvement enables an overall dynamic range larger than $160 \mathrm{~dB}$, which is a record for logarithmic pixels.

\section{Patents}

This work led to the patent application: "Pixel Circuit" European Patent no. EP3354010A1. 
Author Contributions: Conceptualization, methodology, design, validation, formal analysis, investigation, resources, data curation, writing—original draft preparation, A.M.B.; design, writing-review and editing, supervision, funding acquisition, B.C. Both authors have read and agreed to the published version of the manuscript.

Funding: The authors would like to thank the European Commission for funding this research through the EDISON-GA project (Marie Curie Actions, FP7-People Programme).

Acknowledgments: The authors would like to thank Giuseppe di Nicolantonio for the help with the device samples.

Conflicts of Interest: The authors declare no conflict of interest.

\section{References}

1. Freitas, L.M.C.; Morgado-Dias, F. Reference Power Supply Connection Scheme for Low-Power CMOS Image Sensors Based on Incremental Sigma-Delta Converters. Electronics 2021, 10, 299. [CrossRef]

2. Park, K.; Yeom, S.; Kim, S.Y. Ultra-Low Power CMOS Image Sensor with Two-Step Logical Shift Algorithm-Based Correlated Double Sampling Scheme. IEEE Trans. Circuits Syst. I Regul. Pap. 2020, 67, 3718-3727. [CrossRef]

3. Wei, J.; Li, X.; Sun, L.; Li, D. A Low-Power Column-Parallel Gain-Adaptive Single-Slope ADC for CMOS Image Sensors. Electronics 2020, 9, 757. [CrossRef]

4. Gao, Q.; Yadid-Pecht, O. A Low Power CMOS Imaging System with Smart Image Capture and Adaptive Complexity 2D-DCT Calculation. J. Low Power Electron. Appl. 2013, 3, 267-278. [CrossRef]

5. Sukhavasi, S.B.; Sukhavasi, S.B.; Elleithy, K.; Abuzneid, S.; Elleithy, A. CMOS Image Sensors in Surveillance System Applications. Sensors 2021, 21, 488. [CrossRef]

6. Cevik, I.; Huang, X.; Yu, H.; Yan, M.; Ay, S.U. An Ultra-Low Power CMOS Image Sensor with On-Chip Energy Harvesting and Power Management Capability. Sensors 2015, 15, 5531-5554. [CrossRef] [PubMed]

7. Law, M.K.; Bermak, A.; Shi, C. A low-power energy-harvesting logarithmic CMOS image sensor with reconfigurable resolution using two-level quantization scheme. IEEE Trans. Circuits Syst. II Express Briefs 2011, 58, 80-84. [CrossRef]

8. Kim, H.J.; Hwang, S.I.; Kwon, J.W.; Jin, D.H.; Choi, B.S.; Lee, S.G.; Park, J.; Shin, J.; Ryu, S. A delta-readout scheme for low-power CMOS image sensors with multi-column-parallel SAR ADCs. IEEE J. Solid State Circuits 2016, 51, 2262-2273. [CrossRef]

9. Boussaid, F.; Bermak, A.; Bouzerdoum, A. An ultra-low power operating technique for mega-pixels current-mediated CMOS imagers. IEEE Trans. Consum. Electron. 2004, 50, 46-53. [CrossRef]

10. Spivak, A.; Belenky, A.; Fish, A.; Yadid-Pecht, O. Analog Encoding Voltage-A Key to Ultra-Wide Dynamic Range and Low Power CMOS Image Sensor. J. Low Power Electron. Appl. 2013, 3, 27-53. [CrossRef]

11. Schanz, M.; Nitta, C.; Bußmann, A.; Hosticka, B.J.; Wertheimer, R.K. A high-dynamic-range CMOS image sensor for automotive applications. IEEE J. Solid State Circuits 2000, 35, 932-938. [CrossRef]

12. Campos, F.d.S.; Castro, B.A.d.; Swart, J.W. A Tunable CMOS Image Sensor with High Fill-Factor for High Dynamic Range Applications. Eng. Proc. 2020, 2, 79. [CrossRef]

13. El Gamal, A. High dynamic range image sensors. In Proceedings of the Tutorial at International Solid-State Circuits ConferenceInternational Solid State Circuits Conference Tutorials, San Francisco, CA, USA, 7 February 2002; Volume 290.

14. Spivak, A.; Belenky, A.; Fish, A.; Yadid-Pecht, O. Wide-dynamic-range CMOS image sensors-comparative performance analysis. IEEE Trans. Electron Devices 2009, 56, 2446-2461. [CrossRef]

15. Kavadias, S.; Dierickx, B.; Scheffer, D.; Alaerts, A.; Uwaerts, D.; Bogaerts, J. A logarithmic response CMOS image sensor with on-chip calibration. IEEE J. Solid State Circuits 2000, 35, 1146-1152. [CrossRef]

16. Bermak, A.; Kitchen, A. A novel adaptive logarithmic digital pixel sensor. IEEE Photonics Technol. Lett. 2006, 18, 2147-2149. [CrossRef]

17. Choubey, B.; Otim, S.; Aoyama, S.; Joseph, D.; Collins, S. An electronic calibration scheme for logarithmic CMOS image sensor. IEEE J. Sens. 2006, 6, 950-956. [CrossRef]

18. Roy, F.; Tournier, A.; Wehbe-Alause, H.; Blanchet, F.; Boulenc, P.; Leverd, F.; Favennec, L.; Perrot, C.; Pinzelli, L.; Gatefait, M.; et al. Challenges in CMOS-based images. Phys. Status Solidi C 2014, 11, 50-56. [CrossRef]

19. Moon, C.R.; Jung, J.; Kwon, D.W.; Yoo, J.; Lee, D.H.; Kim, K. Application of plasma-doping (plad) technique to reduce dark current of CMOS image sensors. Electron Device Lett. IEEE 2007, 28, 114-116. [CrossRef]

20. Kwon, H.I.; Kwon, O.J.; Shin, H.; Park, B.G.; Park, S.S.; Lee, J.D. The effects of deuterium annealing on the reduction of dark currents in the CMOS APS. IEEE Trans. Electron Devices 2004, 51, 1346-1349. [CrossRef]

21. Seo, M.-W.; Kawahito, S.; Yasutomi, K.; Kagawa, K.; Teranishi, N. A low dark leakage current high-sensitivity CMOS image sensor with STI-less shared pixel design. Electron Devices IEEE Trans 2014, 61, 2093-2097. [CrossRef]

22. Mheen, B.; Song, Y.J.; Theuwissen, A.J.P. Negative offset operation of four-transistor CMOS image pixels for increased well capacity and suppressed dark current. Electron Device Lett. IEEE 2008, 29, 347-349. [CrossRef]

23. Brunetti, A.M.; Musolino, M.; Strangio, S.; Choubey, B. Pixel Design Driven Performance Improvement in 4 T CMOS Image Sensors: Dark Current Reduction and Full-Well Enhancement. IEEE Trans. Electron Devices 2020, 67, 409-412. [CrossRef] 
24. Brunetti, A.M.; Musolino, M.; Choubey, B. Staggered Pixel Layout to Reduce Area and Increase Full Well Capacity in CMOS Image Sensors. IEEE Trans. Electron Devices 2021, 68, 572-577. [CrossRef]

25. Brunetti, A.M.; Choubey, B. A low dark current wide dynamic range CMOS pixel. In Proceedings of the 2016 IEEE International Symposium on Circuits and Systems (ISCAS), Montreal, QC, Canada, 22-25 May 2016; pp. 2523-2526.

26. Joseph, D.; Modelling, S.C. calibration and correction of nonlinear illumination dependent fixed pattern noise in logarithmic CMOS image sensors. In Proceedings of the IMTC 2001, 18th IEEE Instrumentation and Measurement Technology Conference, Rediscovering Measurement in the Age of Informatics (Cat. No.01CH 37188), Budapest, Hungary, 21-23 May 2001; Volume 2, pp. 1296-1301.

27. Sze, S. Semiconductor Devices, Physics and Technology; John Wiley \& Sons: Hoboken, NJ, USA, 1985.

28. Roy, K.; Mukhopadhyay, S.; Mahmoodi-Meimand, H. Leakage current mechanisms and leakage reduction techniques in deepsubmicrometer CMOS circuits. Proc. IEEE 2003, 91, 305-327. [CrossRef] 